\title{
Drug Polymorphism and its Importance on Drug Development Process
}

\author{
Seong Hoon Jeong ${ }^{1 \dagger}$, Yu Seok Youn ${ }^{1}$, Beom Soo Shin ${ }^{2}$ and Eun-Seok Park ${ }^{3}$ \\ College of Pharmacy, Pusan National University, Busan, 609-735, Rep. of Korea \\ College of Pharmacy, Catholic University of Daegu, Gyeongsan, 712-702, Rep. of Korea \\ School of Pharmacy, Sungkyunkwan University, Suwon, 440-746, Rep. of Korea \\ (Received September 7, $2010 \cdot$ Revised October 6, $2010 \cdot$ Accepted October 7, 2010)
}

\begin{abstract}
Polymorphism has been recognized to be a critical issue throughout the drug product development process. Most of solid phase drugs have polymorphism, which has generated a great deal of interest and the field has been evolving rapidly. Preferably, thermodynamically most stable form of a drug substance is selected to obtain consistent bioavailability over its shelf life and various storage conditions. Moreover, it has the lowest potential for conversion from one polymorphic form to another. However, metastable or amorphous forms may be used intentionally to induce faster dissolution rate for rapid drug absorption and higher efficacy. For pharmaceutical industry, polymorphism is one of the key activities in form selection process together with salt selection. This article introduces the main features in the investigation of solid form selection especially polymorphic behavior with thermodynamic backgrounds, physicochemical properties with solubility, dissolution, and mechanical properties, and characterization techniques for proper analysis. The final form can be recommended based on the physicochemical and biopharmaceutical properties and by the processability, scalability and safety considerations. Pharmaceutical scientists especially in charge of formulation need to be well aware of the above issues to assure product quality.
\end{abstract}

Key words - polymorph, solid form, physicochemical properties, drug development, metastable form, amorphous state

Many pharmaceutical active ingredients (APIs) can exist in different solid phases or physical forms; polymorphs, amorphous forms, and solvates. Polymorphism can be defined as the ability of a substance to exist as two or more crystalline phases that have different arrangements and/or conformations of the molecules in the crystal lattice (Grant, 1999; Byrn et al., 1999). Amorphous forms are not crystalline consisting of disordered arrangements of molecules and do not possess a distinguishable crystal lattice. Solvates are crystalline solid adducts containing either stoichiometric or nonstoichiometric amounts of solvent incorporated within the crystal structure. If the incorporated solvent is water, the solvates are also termed as hydrates. Solvates and hydrates are often referred as psuedopolymorphs. Since most of the APIs are crystallized as an initial step for drug development, many drug compounds can exist as crystals with different morphologies by preferential growth of certain crystal faces. Figure 1 shows the various types of solid phases focusing on the internal structure (polymorphism). Different polymorphs of a pharmaceutical solid can have different values in the physicochemical properties of the solid state including crystal packing, bulk properties, ther-

\footnotetext{
†Corresponding Author :

Tel : 82-51-510-2812, E-mail : shjeong@pusan.ac.kr

DOI : 10.4333/KPS.2010.40.S.009
}

modynamic properties, kinetic and mechanical properties, melting point, chemical reactivity, apparent solubility, dissolution rate, optical and electrical properties, vapor pressure, and density. These properties can have a direct impact on the processing of drug substances and the performance of drug products as well, such as dissolution, stability, and bioavailability.

During the drug development process, the lowest energy crystalline polymorph needs to be identified and chosen for development. This step is very important because appearance of another polymorph with lower free energy than the marketed polymorph after approval can be devastating, as previously happened with the HIV protease inhibitor ritonavir (Bauer et al., 2001). Therefore, most of the innovator pharmaceutical companies have been investing significant amount of resources on this solid form selection process early in the new drug development. Moreover, the solid form selection process should cover salt selection together with polymorph, which can be another critical issue for drug development.

The optimal solid form selection of an API can be an important step during the new drug development process. Every institute may have an 'in-house committee' to review every step throughout the drug development procedures including solid form selection, to improve productivity and quality of the selection process with relevant criterion, and to formalize a 


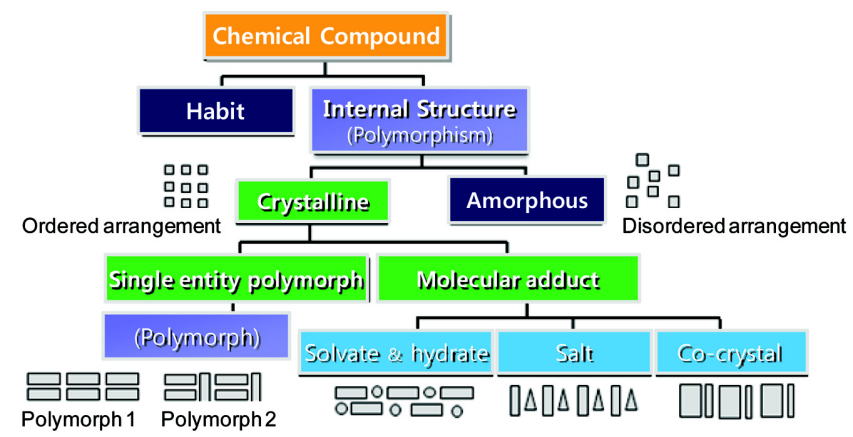

Figure 1. Schematic view of various types of solid forms focusing on the internal structure (polymorphism). Crystalline compounds can have various types of polymorphism including single entity polymorph, solvates, salts, and co-crystals.

project review for every compound that enters from further process. The primary goal of solid form selection can be to identify a crystalline form of the drug substance which can be reproducibly manufactured as a suitable and stable one, physically stable in prototype early formulations, and reproducibly formulated for the IND (investigational new drug application)enabling toxicology studies and the Phase I clinical studies (Ku, 2008).

Recently, the solid form selection studies begin as early as possible and continue until a suitable form is selected and scaled up to produce enough amount of API for the toxicology and early formulation studies. It usually focuses on harmonizing the timelines for form selection with other development activities. The committee may review the activities carried out by working groups that are involved in the form selection, toxicology studies, and project planning activities leading up to the development to arrive at an optimal workflow for making solid form decisions. The key outcome of this review can provide robust scientific criteria for decision-making while accelerating the timelines for the form selection. The multidisciplinary nature of the generation of solid forms and the evaluation of solid state properties necessitates various backgrounds to perform and recommend solid form selection activities, criteria for form selection, and timelines. Figure 2 gives an overview of the general workflow in pharmaceutical industry with project milestone and PK/Tox studies. Even though this review is focusing more on the polymorphism, it will be necessary for the pharmaceutical scientists to be able to see the whole process of the solid form selection including salts and the solid form's physicochemical and biopharmaceutical properties.

As the polymorphism is considered as to influence every stage in the manufacturing and storage of pharmaceutical products, a few regulatory documents (ICH guidelines, 1999; CDER guidelines, 1987; Byrn et al., 1995) address issues

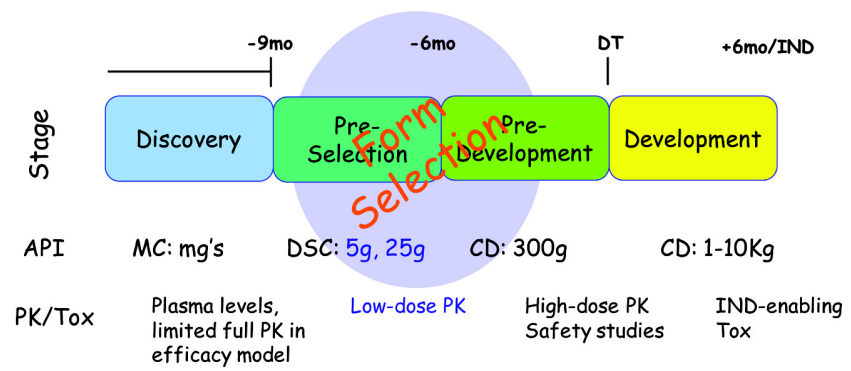

Figure 2. Overview of the general workflow in pharmaceutical industry with project milestone and PK/Tox studies. The form selection is bridging between discovery and development and also facilitating drug development process (modified from $(\mathrm{Ku}, 2008)$ ).

regarding the regulation of polymorphism. Major considerations are focused on monitoring and controlling polymorphs and describing a framework for regulatory decisions regarding the drug substance "sameness" considering the role and impact of polymorphism in pharmaceutical solids. Moreover, the International Conference on Harmonization (ICH) requires investigations and analytical procedures for new drug substances and pharmaceutical products according to a decision tree (ICH guideline, 1999).

\section{Thermodynamic background of polymorphs}

If drug crystals are in liquid or gaseous state, polymorphism does not exist anymore because the structure of solid state no longer exists. However, in the solid state, atoms and molecules are arranged in one of the basic crystal systems: triclinic, monoclinic, orthorhombic, tetragonal, rhombohedral, hexagonal, or cubic. Each crystal system is characterized by unique relationships existing among the crystal axes and the angles between them (Table I). The most widely known example of polymorphism is the carbon element, which can exist in the form of graphite (hexagonal), diamond (cubic), or fullerenes (Figure 3). Paracetamol has different forms; one is monoclinic and another is orthorhombic.

Generally, the relationships between different polymorphs of a drug substance can be explained using Gibbs phase rule (Giron, 2001):

$$
\mathrm{F}+\mathrm{P}=\mathrm{C}+2
$$

where $\mathrm{F}$ is the number of degrees of freedom of the system, which is the number of independent variables that must be fixed to completely determine the system. $\mathrm{P}$ is the number of phases that exist in equilibrium, and $\mathrm{C}$ is the number of components (Giron 2001; Giron and Grant, 2002). In the case of a single drug substance with polymorphism, $\mathrm{C}$ equals one. If one phase (one polymorph) is present, $\mathrm{P}=1$, so $\mathrm{F}=2$. The equation shows that $\mathrm{F}=2$ means that both temperature and pressure may 
Table I. Crystal systems with axial distances and angles

\begin{tabular}{lcc}
\hline \hline \multicolumn{1}{c}{ Crystal system } & Axial distances & Axial angles \\
\hline Triclinic & $\mathrm{a} \neq \mathrm{b} \neq \mathrm{c}$ & $\alpha \neq \beta \neq \gamma$ \\
Monoclinic & $\mathrm{a} \neq \mathrm{b} \neq \mathrm{c}$ & $\alpha=\gamma=90^{\circ} \neq \beta$ \\
Orthorhombic & $\mathrm{a} \neq \mathrm{b} \neq \mathrm{c}$ & $\alpha=\beta=\gamma=90^{\circ}$ \\
Tetragonal & $\mathrm{a}=\mathrm{b} \neq \mathrm{c}$ & $\alpha=\beta=\gamma \neq 90^{\circ}$ \\
Rhombohedral & $\mathrm{a}=\mathrm{b}=\mathrm{c}$ & $\alpha=\beta=90^{\circ} ; \gamma=120^{\circ}$ \\
Hexagonal & $\mathrm{a}=\mathrm{b} \neq \mathrm{c}$ & $\alpha=\beta=\gamma=90^{\circ}$ \\
Cubic & $\mathrm{a}=\mathrm{b}=\mathrm{c}$ &
\end{tabular}

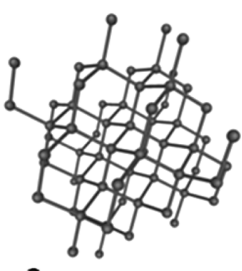

a

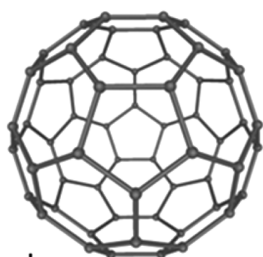

d

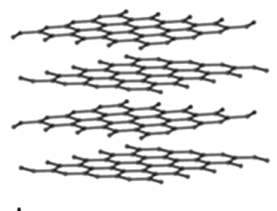

b
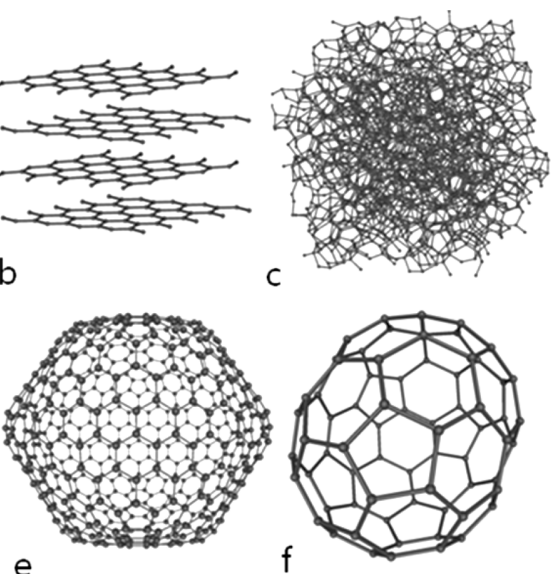

Figure 3. Typical examples of polymorphism with carbon element: (a) diamond (cubic), (b) graphite (hexagonal), (c) amorphous carbon, and (d)-(f) fullerenes $\left(\mathrm{C}_{60}, \mathrm{C}_{540}\right.$, and $\left.\mathrm{C}_{70}\right)$.

be varied without changing the number of phases. If two phases (two polymorphs) are in equilibrium, $\mathrm{P}=2$, so $\mathrm{F}=1$. It means that, at a specific pressure, usually atmospheric pressure, the temperature of the system is fixed at the transition temperature, $T_{\mathrm{t}}$. The phase rule may suggest that only one phase can exist at any given temperature and pressure, except at the transition temperature at a defined pressure, in which case two phases (polymorphs) exist in equilibrium.

According to the phase rule, the process of transformation of one polymorph into another is a phase transition, which may occur at a given pressure by changing the temperature. If the phase transition is reversible, the two polymorphs are enantiotropes, and the energy of the transition of heating is endothermic. If the phase transition is irreversible, the two polymorphs are monotropes, in which case only one form is stable whatever the temperature, and the transformation of the unstable form to the stable one is exothermic. For kinetic reasons, an unstable form may exist for a time outside the region assigned by the phase diagram and the phase rule, and is then termed a metastable form (Lohani and Grant, 2006).

Two types of graphs are usually applied to explain the thermodynamic behavior of polymorphs; energy-temperature (E vs. T) and pressure-temperature (P vs. T) diagrams. $\mathrm{P}$ vs. $\mathrm{T}$ diagrams were introduced briefly in the above. Actually, E vs. $\mathrm{T}$ diagrams were introduced first and the schematic plots of internal energy (U) and Helmholtz free energy (A) vs. temperature were used to represent the phase transformations of crystalline solids. However, the enthalpy of the solids under normal pressure conditions has negligible effects from pressure-volume energy. The ability of a system to perform work

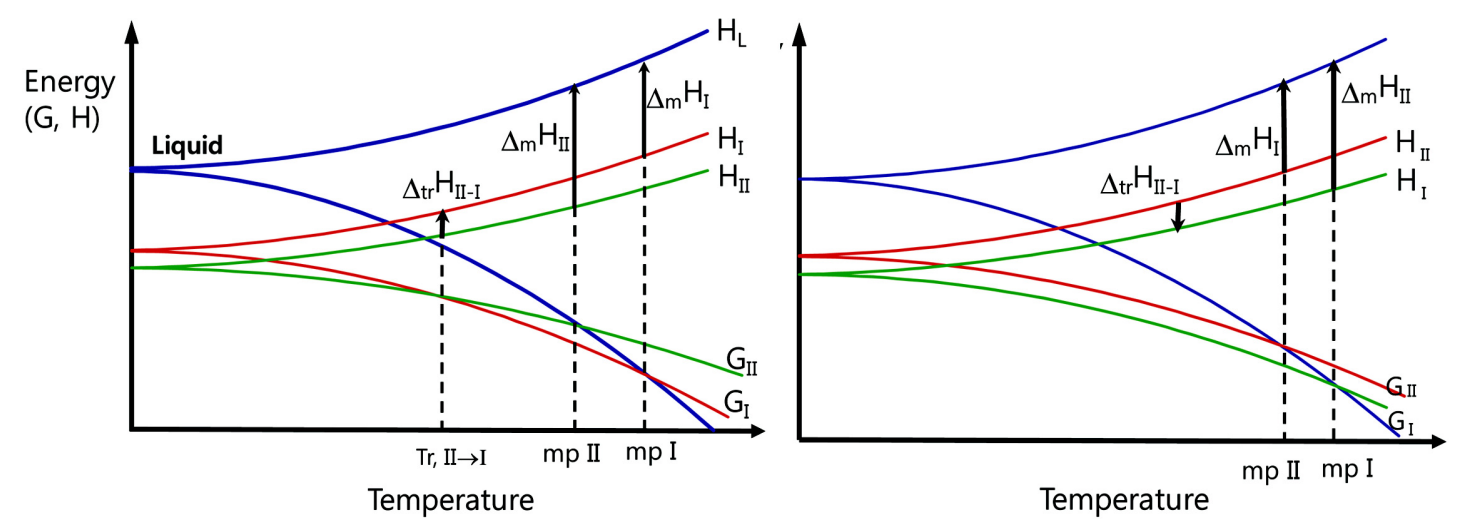

Figure 4. Energy (Gibbs free energy and enthalpy)-temperature plots for an enantiotropic and a monotropic system. If the two curves intersect below the melting point of each form, then these polymorphs are said to be enantiotropic. If not, they are monotropic and the higher melting form is always the thermodynamically stable form. 
and to undergo a spontaneous change at ambient pressure is measured by the Gibbs free energy, G.

$$
\begin{aligned}
& \mathrm{H}=\mathrm{U}+\mathrm{PV} \approx \mathrm{U} \\
& \mathrm{G}=\mathrm{H}-\mathrm{TS} \approx \mathrm{U}-\mathrm{TS}=\mathrm{A}
\end{aligned}
$$

The thermodynamic relationship between two polymorphic phases can be plotted as the Gibbs free energy vs. temperature for each form (Figure 4). If the two curves intersect below the melting point of each form, a reversible solid-solid transformation occurs at the temperature $\left(T_{\mathrm{t}}\right)$ of the intersection. These polymorphs are said to be enantiotropic. At temperatures below $T_{\mathrm{t}}$, polymorph $\mathrm{A}$ has the lower free energy than $\mathrm{B}$, so the polymorph $\mathrm{A}$ is the thermodynamically stable form. However, at temperatures above $T_{\mathrm{t}}$ polymorph B is stable because its free energy is lower than that of polymorph A. In case of monotropic system, the higher melting form is always the thermodynamically stable form.

\section{Effects of polymorphism on bioavailability}

Bioavailability of many drugs can be dependent on polymorphs affecting solubility and absorption rate (Singhhal and Curatolo, 2004). Typical examples are chloramphenicol palmitate, mefenamic acid, oxytetracycline, and carbamazepine. Absorption of chloramphenicol palmitate polymorph B was significantly greater than polymorph $\mathrm{A}$ in humans (Aguiar et el., 1967). Chloramphenicol peak serum levels showed linear relationship to the percentage of Form B in Form A/Form B mixtures. Moreover, in vitro hydrolysis of the prodrug by pancreatin was confirmed to be polymorph-dependent, with significantly more hydrolysis of polymorph $\mathrm{B}$ compared to polymorph A. Moreover, Form B dissolved faster than Form A with higher solubility (Aguiar et el., 1969). This solubility difference might result in the difference in ester hydrolysis rates, and the difference in oral absorption.

Recently, six bulk oxytetracycline samples were compared and four of these contained one polymorph while the other two contained a different polymorph (Form A) (Liebenberg et al., 1999). Tablets prepared from the Form A dissolved significantly more slowly than the. The Form A tablets exhibited $\sim 55 \%$ dissolution at $30 \mathrm{~min}$, while the others showed complete $(\sim 95 \%)$ dissolution at $30 \mathrm{~min}$.

Three out of 53 batches of generic carbamazepine tablets were recalled due to clinical failures and dissolution changes (Meyer et al., 1992). In vitro dissolution test was performed in water containing $1 \%$ sodium lauryl sulfate. It revealed that two of the batches dissolved more slowly than the innovator product, and one batch dissolved more quickly. While the innovator product gave $\sim 95 \%$ dissolution in $90 \mathrm{~min}$ in the medium, the slower generic batches gave $\sim 35 \%$ and $75 \%$ dissolution. In humans, the generic batches gave mean relative AUCs (area under the curve) of $60-113 \%$, with the same rank order observed in the in vitro dissolution behavior. It was suggested that moisture uptake during storage and particle size differences may have been involved in the irreproducible behavior of the generic tablets of the. It is also known that anhydrous carbamazepine converts to the dihydrate quickly when the anhydrous form is suspended in water (Young and Suryanarayanan, 1991). Therefore, a significant solubility difference between two polymorphs may cause a difference in solubility and oral drug absorption rate resulting in bioavailability change, and hence every scientist needs to be well aware of the polymorphic backgrounds when to develop oral drug products.

\section{Solubility and dissolution}

Polymorphs of a drug substance can have different apparent aqueous solubility and dissolution rate. If such differences are sufficiently large, bioavailability would be changed. Therefore, it will be difficult to formulate a bioequivalent drug product using a different polymorph.

Polymorphic differences and transformation that result in different apparent solubility and dissolution rate are generally detected by dissolution testing. Traditionally, aqueous solubility of drugs is determined using the equilibrium solubility method that involves suspending an excess amount of a solid drug in a selected aqueous medium. However, the equilibrium solubility may not be suitable to obtain the solubility of a metastable form, because the metastable form may convert to the stable form during the experiment. When the solubility of metastable forms of a drug substance cannot be determined by the equilibrium method, the intrinsic dissolution method might be useful to deduce the relative solubility of metastable forms (6).

As introduced in the previous example of chloramphenicol palmitate (Aguiar et el., 1967), a change in the energy of the interactions as a solute dissolves can be expressed as the enthalpy of solution, $\Delta H_{\text {sol }}$, while the standard free energy change, $\Delta G^{\theta}$, is related to the solubility $c_{s}$.

$$
\Delta G_{\text {sol }}^{\ominus}=-R T \ln c_{s}
$$

where $R$ is the gas constant and $T$ is the absolute temperature (Giron and Grant, 2002). According to the van't Hoff equation, the logarithm of the equilibrium constant (solubility product) is a linear function of the reciprocal of the absolute temperature, as following,

$$
\begin{aligned}
& \ln K_{\mathrm{s}}=\ln K_{0}-\Delta H_{\mathrm{sol}} / R T \\
& \text { or } K_{\mathrm{s}}=K_{0} \cdot \mathrm{e}^{-\Delta H \mathrm{sol} / R T}
\end{aligned}
$$



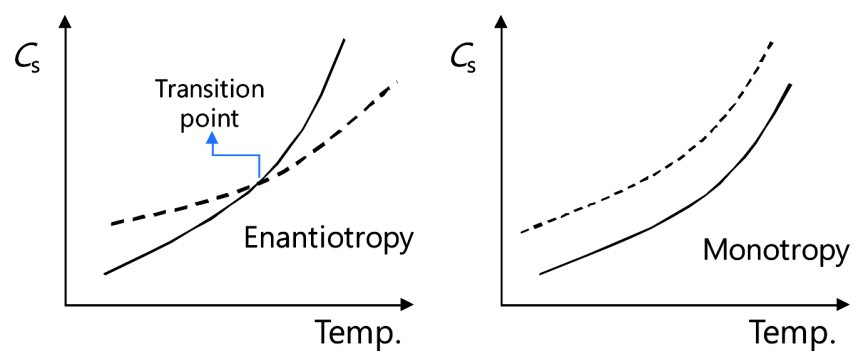

Figure 5. Plots of solubility $\left(C_{\mathrm{s}}\right)$ vs. temperature curves for enantiotropic and monotropic systems.

The solubility of polymorphs is related to their thermodynamic activity, to the escaping tendency of their molecules, and hence to their melting point. The thermodynamically stable form at a given temperature and pressure is the form with the lowest free energy and the poorest solubility.

For each polymorph, the van't Hoff equation can be applied as following.

$$
\ln c_{s}=-\Delta H_{\mathrm{sol}} / R T+c
$$

where $c$ is a constant. The solubility curves obtained are shown in Figure 5. In the case of enantiotropy, there is a transition point at which the solubility of the two polymorphs is identical. However, in the case of monotropy, the curves do not intersect. If a solvent-mediated transition occurs, it would result in spontaneous precipitation of the thermodynamically stable form. Plotting $\ln c_{s}$ vs. 1/T for each modification allows the determination of the transition point and the calculation of $\Delta H_{\text {sol }}$ for each modification. The difference of $\Delta H_{\text {sol }}$ between the two forms can be the transition enthalpy. In the case of different hydrates, different transition points can be observed. This solubility-temperature approach is frequently utilized for determining the relationship between the polymorphs of anhydrous forms and for solvates or hydrates. Generally, the solubility in water increases in the rank order: hydrate $<$ anhydrate $<$ solvated form, but exceptions still exist, especially when the transition temperature lies below the ambient temperature (Giron and Grant, 2002).

\section{Stability and mechanical properties}

Polymorphs of a pharmaceutical solid may have different physicochemical properties (9). The most stable polymorphic form of a drug substance is often selected because it has the lowest potential for conversion from one polymorphic form to another. However, metastable or amorphous forms may be used intentionally to enhance the bioavailability. An amorphous form is thermodynamically less stable than any crystalline forms with the higher molecular mobility and reactivity.

The more thermodynamically stable polymorph is recog- nized to be more chemically and/or physically stable than a metastable one due to the higher crystal packing density of the thermodynamically favored polymorph. However, other factors, such as optimized orientation of molecules, and hydrogen bonds and non-hydrogen bonds in the crystal lattice play important roles. Relatively small changes in crystal packing may lead to significant differences in the crystal packing density and chemical reactivity of two polymorphs, as indomethacin polymorphs (Chen et al., 2002).

Polymorphism can also affect the mechanical properties of drug particles impacting the manufacturability and physical attributes of tablets. For example, polymorphs of paracetamol (Di Martino et al., 1996; Nichols and Frampton, 1998; Beyer et al., 2001; Joiris et al., 1998), sulfamerazine (Sun and Grant, 2001), carbamazepine (Roberts et al., 2000; Otsuka et al., 1999), and phenylbutazone (Summers et al., 1976) have been addressed different mechanical properties. A common effect of polymorphism can be the change of powder flow due to the difference in particle morphology of polymorphs. Polymorphs with needle- or rod-shaped particles may have poor flowability compared to polymorphs with low aspect ratio. The effect of polymorphism on other mechanical properties including hardness, compressibility, and bonding strength is more complicated.

In addition, polymorphic conversions of some drug substances are possible when exposed to a wide range of manufacturing processes (Byrn et al., 1999). Milling/micronization operations may result in polymorphic form conversion. In the case of wet granulation processes, where the usual solvents are water, one may encounter a variety of interconversions between anhydrates and hydrates, or between different hydrates. Spray-drying processes have been shown to produce amorphous drug substances. However, phase conversions should not be an issue if they occur consistently and are controllable.

\section{Characterization of solid forms in drug development process}

A number of methods have been employed for characterizing solid forms throughout the drug development process. The definitive criterion for the existence of polymorphism is via demonstration of a nonequivalent crystal structure, usually by comparing the $\mathrm{x}$-ray diffraction patterns. Thermal analysis, such as differential scanning calorimetry (DSC) and thermogravimetric analysis (TGA), can be used to obtain thermodynamic information, including phase changes, and to deduce whether each isolated form is a solvate or anhydrate. These thermal methodologies can distinguish between enantiotropic 
and monotropic systems. Polarizing optical microscopy and thermomicroscopy are very useful but simple tools. Solid state nuclear magnetic resonance (NMR), infrared absorption, and Raman spectroscopy are used to study crystal structures. Microscopy, thermal analysis methodology, and solid state NMR are generally considered as sources of supporting information.

During the drug development procedures, form selection might be initiated with the evaluation of the physicochemical properties and dosing vehicle options for the discovery compounds. Usually, the discovery candidates are synthesized in small scales, typically from $\mathrm{mg}$ to $\mathrm{g}$ scale. Representative batches need to be characterized for purity, crystal form, solubility, and solution stability simulating in vivo conditions. Drug candidates that are nominated for advancement to the later stages are usually scaled close to $100 \mathrm{~g}$ batch size. A representative batch needs to be evaluated for a full physical and chemical profile. This includes evaluation of purity, crystallinity, residual solvents, hygroscopicity, equilibrium solubility, solution state stability, log P measurements, solid state stability, and plasma protein binding.

\section{Screening of solid forms for development}

\section{A. Free acid/base polymorph screening}

The evaluation of any candidates should begin with a review of the physical characterization. When stable free base or free acid species can be prepared, the free species need to be crystallized and evaluated their possible polymorphs. The main purpose of the screening is to establish the range of crystalline forms and evaluate their properties such that a form could be selected for development before the completion of the large scale batch synthesis. Screening for solid forms can be carried out as a function of solvent, temperature, concentration, and nucleation methods. For initial screening, these variables should be open ended to give a broad exploration of "form space". Subsequent screening could be limited to pharmaceutically acceptable solvents, focusing on hydrates and neat forms.

High-throughput form screening technology can be proposed, and this technology relies on high throughput sample preparation, equilibration, and automated analysis with optical microscopy and DSC/TGA. It will allow rapid evaluation with $24 \mathrm{hr}$ equipment operation. The hits obtained from such a screening can be further characterized.

\section{B. Preparation and evaluation of salt forms}

Some institutes preferred to select a crystalline non-salt API for development, but in some cased it may be necessary to evaluate a salt of the API. Typically 5-10 g of API will be used for this screen. The following conditions will be used to trigger the decision for salt screening:

- Chemical instability of the free base or free acid

- Inability to obtain crystalline free base or free acid with acceptable purity

- Physical instability by low melting temperature, moisture absorption, or other storage considerations

Initial salt screening will focus on the preparation of milligram quantities of salts using common solvents and several counter ions. Preferably following counter ions will be advantageous due to the purity and cost benefits; acetic acid, benzoic acid, citric acid, fumaric acid, hydrochloric acid, tartaric acid, malic acid, maleic acid, phosphoric acid, sulfuric acid, succinic acid, and lactic acid. Once a few salt leads are identified, it may be necessary to carry out a separate study to screen for polymorphs of the salts as above.

\section{Characterization of solid forms}

Once a procedure for obtaining a solid is discovered, it is important to characterize the phase composition. Many physicochemical properties such as thermal behavior, apparent solubility, behavior on drying, moisture uptake, and slurry behavior of a mixture can display significant departures from those of a pure phase, even if the impurity phase is present in relatively minor amounts. It is also a source of considerable confusion when analytical data, intended for use as a fingerprint for the phase, are actually obtained on a mixture. The characterization of a solid might proceed in a two-step process, a preliminary analysis, possibly followed by a more detailed analysis. The sample requirements for various analyses need to be considered carefully. Traditional techniques (Microscopy, TGA, DSC) are the most useful in this preliminary work.

- Microscopy using polarized light generally reveals if the sample is crystalline

- TGA detects the presence of volatile components, indicating the possible presence of lattice solvents, and/or decomposition

- DSC detects thermal events (desolvation/evaporation/boiling, sublimation, melting, recrystallization, and/or decomposition) and provides information on phase composition

- Additional techniques includes IR, Raman, SEM, and hot stage microscopy

At the completion of the preliminary analysis there will be normally sufficient information to determine whether the sample contains a new crystalline phase. If no new crystal phases are present, any more characterization may be unnecessary. However, if a new crystal phase is detected, more extensive 
characterization is required. For example, the preliminary study provides no guarantee that a mixture of phases can be distinguished from a single phase. Moreover, the composition of a given solid phase (e.g. counter-ion stoichiometry, lattice solvent), as opposed to the composition of the sample, which may be heterogeneous, is not adequately addressed by these techniques. In every case, a PXRD (powder x-ray diffraction) pattern of any crystalline material must be obtained for comparison at a later time. If necessary, more specialized experiments will be necessary including variable temperature PXRD (VTPXRD), hot-stage microscopy, and moisture balance measurements. These techniques are useful in resolving many ambiguities that can persist after preliminary characterization.

- Hot stage microscopy can detect phase conversions during heating/drying

- VTPXRD can identify the new phases that occur during heating/drying

- The moisture balance can detect and characterize the hydrates' behavior

Single crystal X-ray structure determination can provide valuable information. Single crystal data may be also inferred from high-resolution powder diffraction data by the use of existing computational chemistry procedures, and vice versa. Other techniques can be useful in the early stages. For dried powders, HPLC, GC and solution NMR can be used on a redissolved sample to detect solvents of crystallization and the presence of organic counter-ions. ICP-OES can be used to detect inorganic counter-ions. Raman, NIR and IR spectroscopy are also useful in solid state characterization, especially if the spectra of the pure crystal phases are available for reference. When the available resources are not enough to undertake analysis of all forms encountered during the experiments, the following types of forms should be given priority:

- All neat polymorphic forms of the free acid/base

- All potentially important solvates

- All polymorphic forms of the chosen salt

- All potentially important solvates of the chosen salt

Since neither the final process nor the details of the formulation are generally known at the time of form investigations, it will be critical for the scientists to judge the issues carefully.

\section{Considerations on scalability and chemical processing}

The candidate form must be amenable for scale-up process. Ideally, the final form is the thermodynamically most stable in the range of the processing conditions for crystallization and subsequent handling. The relative stability of different polymorphs and their crossover points with respect to the pro- cessing conditions during the process development need to be investigated. Significant efforts need to be made to determine the relative stability of various polymorphs by comparing solubility, heat of fusion, melting point, and stability by mixture slurrying experiments. If direct comparison of thermodynamic stability is not possible, as in the case of comparing a neat form with a hydrate, then it has to be demonstrated which form can be made reproducibly in the solvents used in the process. A metastable form might be avoided if possible, unless the metastable form offers significant advantage in other respects such as bioavailability and drug product manufacturing.

Knowledge generated in early form selection stage is particularly relevant to process development when the candidate is a metastable form. In these cases, conditions for polymorph conversion to a more stable form should be under control in each step of the process. The final step of process chemistry will be dynamic and flexible at the early stage. Upon selection of an interim form, additional process knowledge should be prepared as followings;

- chemical stability

- processing conditions such as reagents, solvents, reaction conditions, and drying

- engineered crystallization protocols (cubic crystallization, impinging jet, sonication)

- applicability of in-process analytical tools (in-line Raman, near IR)

- particle size processing method (milling, micronization)

- storage/handling of the material

Ideally, the requisite solid form should be integrated into the manufacturing process without adding additional processing steps at the end. The goal is to keep the entire process as simple and elegant as possible to maximize overall yield and purity. Other considerations from the process standpoint will be;

- crystal habit

- filterability

- bulk properties

- compatibility of materials of construction with counter-ions

- use of "green" solvents

\section{Assessing suitability for drug product development}

The desirable properties of a solid form of the drug substance are dependent on the target product, oral or parenteral dosage forms.

A. Oral dosage form: general criteria considered in evaluating the suitability of a form for solid dosage form development are: 
- Crystallinity

- Aqueous solubility

- Hygroscopicity

- Physicochemical solid-state stability

- Presence of polymorphs/pseudopolymorphs

- Identification of the thermodynamically stable form(s)

- Enantiotropic versus monotropic behavior

- Hydrates versus anhydrates

- If a metastable form must be selected and conversion affects drug performance, the boundary conditions for storage and handling of this form must be identified.

- Propensity for phase transformations in prototype formulations

- If necessary, an exposure study in animals can be performed to compare the relative bioavailability of the free $\mathrm{acid} / \mathrm{base}$ with the corresponding solvate/hydrate or salt form.

- The powder properties such as bulk density, compressibility, and compactability, may be considered, if necessary.

B. Parenteral dosage form: additional criteria considered in assessing the suitability of a form for parenteral dosage form development are:

- Solution stability (potential influence of counter-ion on stability)

- Solubility as a function of $\mathrm{pH}$

- Presence of solvent(s) of crystallization in the API and their level of acceptability in the final dosage form.

C. Other delivery routes (buccal, pulmonary): special considerations need to be handled on a case-by-case basis.

\section{Conclusion: final form recommendation}

The decision making for final form selection should be driven by the physicochemical and biopharmaceutical properties, and by the processability, scalability and safety considerations. In most cases, a neat form with acceptable solidstate properties, presents fewer issues during development. The in-house committee maintains an active "project milestones" to monitor the form status throughout the pre-clinical and clinical studies and is responsible for assigning a particle size recommendation for the recommended final form.

\section{Acknowledgements}

This study was supported by a grant of the Korean Health Technology R\&D Project, Ministry for Health, Welfare \& Family Affairs, Republic of Korea. (A092018)

\section{References}

Aguiar, A.J., Krc, J., Kinkel, A.W., Samyn, J.C., 1967. Effect of polymorphism on the absorption of chloramphenicol from chloramphenicol palmitate. J. Pharm. Sci. 56, 847-853.

Aguiar, A.J., Zelmer, J.E, 1969. Dissolution behavior of polymorphs of chloramphenicol palmitate and mefanamic acid. J. Pharm. Sci. 58, 983-987.

Beyer, T., Day, G.M., Price, S.L., 2001. The prediction, morphology and mechanical properties of the polymorphs of paracetamol. J. Am. Chem. Soc. 123, 5086-5094.

Byrn, S., Pfeiffer, R., Ganey, M., Hoiberg, C., Poochikian, G., 1995. Pharmaceutical solids: A strategic approach to regulatory considerations. Pharm. Res. 12, 945-954.

Byrn, S.R., Pfeiffer, R.R., Stowell, J.G., 1999. Solid-state chemistry of drugs (2nd ed.). SSCI, Inc., West Lafayette, Indiana.

Bauer, J., Spanton, S., Henry, R., Quick, J., Dziki, W., Porter, W., Morris, J., 2001. Ritonavir: an extraordinary example of conformational polymorphism. Pharm. Res. 18, 859-866.

Center for Drug Evaluation and Research Guidance: Submitting Supporting Documentation in Drug Applications for the Manufacture of Drug Substances, February 1987.

Chen, X., Morris, K.R., Griesser, U.J., Byrn S.R., Stowell, J.G., 2002. Reactivity differences of indomethacin solid forms with ammonia gas. J. Am. Chem. Soc. 124, 15012-15019.

Di Martino, P., Guyot-Hermann, A.M., Conflant, P., Drache, M., Guyot, J.C., 1996. A new pure paracetamol for direct compression: the orthorhombic form. Int. J. Pharm. 128, 1-8.

Giron, D., Grant, D.J.W., 2002. Evaluation of solid-state properties of salts. In Stahl, P.H., Wermuth, C.G. (eds.) Handbook of pharmaceutical salts: properties, selection, and use. WileyVCH, Surich, Switzerland, pp 41-81.

Giron, D., 2001. Investigation of polymorphism and pseudo-polymorphism in pharmaceuticals by combined thermoanalytical techniques. J. Therm. Anal. Calorim., 64, 37-60.

Grant, D.J.W., 1999. Theory and origin of polymorphism. In H. G. Brittain (ed.) Polymorphism in Pharmaceutical Solids. Marcel Dekker, Inc., New York, pp. 1-34.

International Conference on Harmonization Q6A Guideline: Specifications for New Drug Substances and Products: Chemical Substances, October 1999.

Joiris, E., Di Martino, P., Berneron, C., Guyot-Hermann, A.M., Guyot, J.C., 1998. Compression behavior of orthorhombic paracetamol. Pharm. Res. 15, 1122-1130.

$\mathrm{Ku}$, M.S., 2008. Use of the Biopharmaceutical Classification System in Early Drug Development. AAPS J. 10, 208-212.

Liebenberg, W., de Villiers, M., Wurster, D.E., Swanepoel, E., Dekker, T.G., Lotter, A.P., 1999. The effect of polymorphism on powder compaction and dissolution properties of chemically equivalent oxytetracycline hydrochloride powders. Drug Dev. Ind. Pharm. 25, 1027-1033.

Lohani, S., Grant, D.J.W., 2006. Thermodynamics of polymorph. In Hilfiker, R. (ed.) Polymorphism in the pharmaceutical industry. Wiley-VCH Verlag GmbH \& Co. KGaA, Weinheim, 
Germany, pp 21-42.

Meyer, M.C., Straughn, A.B., Jarvi, E.J., Wood, G.C., Pelsor, F.R., Shah, V.P., 1992. The bioinequivalence of carbamazepine tablets with a history of clinical failures. Pharm. Res. 9, 16121616.

Nichols, G., Frampton, C.S., 1998. Physicochemical characterization of the orthorhombic polymorph of paracetamol crystallized from solution. J. Pharm. Sci. 87, 684-693.

Otsuka, M., Hasegawa, H., Matsuda, Y., 1999. Effect of polymorphic forms of bulk powders on pharmaceutical properties of carbamazepine granules. Chem. Pharm. Bull. 47, 852-856.

Roberts, R.J., Payne, R.S., Rowe, R.C., 2000. Mechanical property predictions for polymorphs of sulphathiazole and carbam- azepine. Eur. J. Pharm. Sci. 9, 277-283.

Singhhal, D., Curatolo, W., 2004. Drug polymorphism and dosage form design: a practical perspective. Adv. Drug Del. Rev. 56, 335-347.

Summers, M.P., Enever, R.P., Carless, J.E., 1976. The influence of crystal form on the radial stress transition characteristics of pharmaceutical materials. J. Pharm. Pharmacol. 28, 89-99.

Sun, C., Grant, D.J.W., 2001. Influence of crystal structure on the tableting properties of sulfamerazine polymorphs. Pharm. Res. 18, 274-280.

Young, W.W.L., Suryanarayanan, R., 1991. Kinetics of transition of anhydrous carbamazepine to carbamazepine dihydrate in aqueous suspensions. J. Pharm. Sci. 80, 496-500. 